EPJ Web of Conferences 20, 03001 (2012)

DOI: $10.1051 /$ epjconf/20122003001

(C) Owned by the authors, published by EDP Sciences, 2012

\title{
Charmonium spectrum at finite temperature from a Bayesian analysis of QCD sum rules
}

\author{
Philipp Gubler ${ }^{1}$, Kenji Morita ${ }^{2}$, and Makoto Oka ${ }^{1}$ \\ 1 Department of Physics, Tokyo Institute of Technology, Meguro, Tokyo 152-8551, Japan \\ 2 Yukawa Institute for Theoretical Physics, Kyoto University, Kyoto 606-8502, Japan
}

\begin{abstract}
Making use of a recently developed method of analyzing QCD sum rules, we investigate charmonium spectral functions at finite temperature. This method employs the Maximum Entropy Method, which makes it possible to directly obtain the spectral function from the sum rules, without having to introduce any strong assumption about its functional form. Finite temperature effects are incorporated into the sum rules by the change of the various gluonic condensates that appear in the operator product expansion. These changes depend on the energy density and pressure at finite temperature, which are extracted from lattice QCD. As a result, $J / \psi$ and $\eta_{c}$ dissolve into the continuum already at temperatures around $1.0 \sim 1.1 T_{c}$.
\end{abstract}

\section{Introduction}

Ever since the establishment of QCD as the theory of the strong interaction, the charmonium system has been used as a probe of both its perturbative and non-perturbative dynamics [1]. Moreover, charmonia in hot or dense matter have also been investigated intensively in both theoretical and experimental studies. To a large degree, this interest is related to the suggestion that in the color-deconfined medium with a temperature above $T_{c}, J / \psi$ will melt due to the color Debye screening, and thus serve as a signal for formation of quark-gluon plasma [2,3]. A full understanding of the phenomena, which are relevant for the experimental observation of $J / \psi$ suppression is however not yet obtained and further studies are necessary [4].

In connection with these efforts, it is important to make reliable predictions form first principles of QCD. This is a difficult task, but quite a lot of progress in this direction has been made during the last decade [5], mainly due to the developments of lattice QCD in combination with the maximum entropy method (MEM) [6-9]. From these studies, the picture emerged that the lowest charmonium states $\left(J / \psi\right.$ and $\left.\eta_{c}\right)$ survive up to temperatures around $1.5 T_{c}$ or even higher. It is one of our goals in the present study to examine these results from the viewpoint of QCD sum rules.

The method of QCD sum rules, developed by Shifman et al. [10] more than thirty years ago, provides us with a tool for exploring the properties of hadrons not only in vacuum but also at finite temperature. The extension of the the method to finite temperature was worked out in $[11,12]$ and essentially relies on the assumption that all the finite temperature effects can be included into the changing values of the condensates. Within this approach, the the charmonium system was recently studied [13-16], and evidence for a substantial mutation of the corresponding spectral function just above $T_{c}$ was found. In these studies, it was however not possible to discuss the possible melting of the charmonium states, because a relativistic Breit-Wigner ansatz was used to parametrize the spectral function at all investigated temperatures and its dominance over the dispersion integral was required to validate the analysis procedure. The second goal of the present study is to identify the actual nature of this change of the spectral function around $T_{c}$.

a e-mail: phil@th.phys.titech.ac.jp

This is an Open Access article distributed under the terms of the Creative Commons Attribution-Noncommercial License 3.0, which permits unrestricted use, distribution, and reproduction in any noncommercial medium, provided the original work is properly cited. 


\section{EPJ Web of Conferences}

For this purpose, we use MEM, which was recently proposed to be a useful analysis method for QCD sum rules [17]. This approach has the advantage that it is not necessary to introduce any strong assumptions on the functional form of the spectral function, such as the Breit-Wigner pole used in [13-16]. It thus becomes possible to study the sum rules without prejudice, and obtain the most probable spectral function from considerations based on Bayesian statistics. This method has already been successfully applied to the $\rho$-meson [17] and nucleon [18] channels.

This proceedings article is based on [19] and is organized as follows. In Section 2, the charmonium sum rules at zero and finite temperature are explained. In Section 3, the details of the MEM analysis are described, while in the results are outlined in Section 4. Finally, the conclusions are given in Section 5 .

\section{Formalism}

The starting point of QCD sum rules at finite temperature is the time ordered correlator, evaluated in a hot medium:

$$
\Pi^{\mathrm{J}}(q)=i \int d^{4} x e^{i q x}\left\langle T\left[j^{\mathrm{J}}(x) j^{\mathrm{J}}(0)\right]\right\rangle_{T}
$$

In the present study, $j^{\mathrm{J}}(x)$ stands for $\bar{c} \gamma_{\mu} c(x)$ in the vector $(\mathrm{V})$ and $\bar{c} \gamma_{5} c(x)$ in the pseudoscalar (PS) channel. The definition of the expectation value $\langle O\rangle_{T}$ is $\langle O\rangle_{T} \equiv \operatorname{Tr}\left(e^{-H / T} O\right) / \operatorname{Tr}\left(e^{-H / T}\right)$. For the current work, we will set the spatial momentum of the charmonium system relative to the thermal medium to be $\mathbf{0}$, therefore $q^{\mu}=(\omega, \mathbf{0})$. In this situation, there is only one independent component in the correlator of the vector channel. For the following analysis, the dimensionless functions $\tilde{\Pi}^{\mathrm{V}}\left(q^{2}\right) \equiv$ $\Pi_{\mu}^{\mu, \mathrm{V}}(q) /\left(-3 q^{2}\right)$ and $\tilde{\Pi}^{\mathrm{PS}}\left(q^{2}\right) \equiv \Pi^{\mathrm{PS}}(q) / q^{2}$ will be employed.

Using a dispersion relation, one can connect the values of the correlator calculated in the deepEuclidean region $\left(q^{2} \equiv-Q^{2} \ll 0\right)$ to an integral of the spectral function $\rho^{J}(\omega)$. After applying the Borel transform, one arrives at the following expression:

$$
\mathcal{M}^{J}\left(M^{2}\right)=2 \int_{0}^{\infty} d \omega \omega e^{-\omega^{2} / M^{2}} \rho^{J}(\omega)
$$

Here, $M$ is the Borel mass. In this equation, the left-hand side can be calculated analytically using the operator product expansion (OPE). To obtain the spectral function $\rho^{J}(\omega)$ one therefore has to invert the integral of Eq. (2). This is, however, an ill-posed problem and can not be solved rigorously. For this purpose, we will use the MEM analysis, which will be explained in the next section.

The result of the OPE can be formally given as

$$
\mathcal{M}^{\mathrm{J}}\left(M^{2}\right)=e^{-v} A^{\mathrm{J}}(v)\left[1+\alpha_{s}(v) a^{\mathrm{J}}(v)+b^{\mathrm{J}}(v) \phi_{b}(T)+c^{\mathrm{J}}(v) \phi_{c}(T)+d^{\mathrm{J}}(v) \phi_{d}(T)\right],
$$

where $v$ stands for the dimensionless parameter $4 m_{c}^{2} / M^{2}, m_{c}$ being the charm quark mass. The first two terms in this equation are the leading order perturbative term and its first order $\alpha_{s}$-correction. The third and fourth terms consist of the scalar and twist- 2 gluon condensates of mass dimension 4: $\phi_{b}(T)=$ $\frac{4 \pi^{2}}{9\left(4 m_{c}^{2}\right)^{2}} G_{0}$ and $\phi_{c}(T)=\frac{4 \pi^{2}}{3\left(4 m_{c}^{2}\right)^{2}} G_{2}$, where $G_{0}=\left\langle\frac{\alpha_{s}}{\pi} G_{\mu \nu}^{a} G^{a \mu \nu}\right\rangle_{T}$ and $G_{2}$ is defined as $\left\langle\frac{\alpha_{s}}{\pi} G^{a \mu \sigma} G_{\sigma}^{a v}\right\rangle_{T}=$ $\left(u^{\mu} u^{v}-\frac{1}{4} g^{\mu v}\right) G_{2}$, with $u^{\mu}$ expressing the four velocity of the heat bath. For the detailed expressions of the Wilson coefficients of these terms, see $[20,16]$. To get an idea on the validity of the OPE convergence, we include also the fifth therm, containing the scalar gluon condensate of dimension 6 , $\phi_{d}(T)=\frac{1}{\left(4 m_{c}^{2}\right)^{3}}\left\langle g^{3} f^{a b c} G_{\mu}^{a v} G_{v}^{b \lambda} G_{\lambda}^{c \mu}\right\rangle_{T}$. The Wilson coefficient of this term has been calculated in [21]. It has to be mentioned here that in the vector channel, there is actually still another term present in the OPE, the so-called scattering term, which is a constant and stems from a pole at $\omega=0$ in $\rho^{\mathrm{V}}(\omega)$. This term has also been discussed in lattice studies [22] and considerably complicates the analysis. We therefore eliminate it by taking the derivative with respect to $v$ and analyzing the resulting sum rule in this channel.

When going to finite temperature $T$, it was argued in [12] that as long as $T$ lies below the separation scale of the OPE $(\sim 1 \mathrm{GeV})$, all finite temperature effects should be included into the various 
condensates appearing in the OPE. We will follow the same approach and consider the influence of the temperature of the gluonic condensates as the only effect to be considered in comparison with the zero temperature case. The question is now how to obtain the values of the condensates at finite $T$. A possible solution to this problem was proposed in $[13,14]$, where the expressions of the energy momentum tensor using both gluonic operators and thermodynamic quantities such as the energy density $\epsilon(T)$ and pressure $p(T)$ were compared. From this comparison, the following relations between the dimension 4 gluonic condensates and $\epsilon(T)$ and $p(T)$ were obtained:

$$
\begin{aligned}
G_{0} & =G_{0}^{\mathrm{vac}}-\frac{8}{11}[\epsilon(T)-3 p(T)], \\
G_{2} & =-\frac{\alpha_{s}(T)}{\pi}[\epsilon(T)+p(T)] .
\end{aligned}
$$

The functions $\epsilon(T), p(T)$ and $\alpha_{s}(T)$ were then extracted from quenched lattice QCD data [23,24]. In this work, we use the same numerical values for the $T$ dependent part of $G_{0}$ and $G_{2}$ as in [16]. It should be noted that due to the sudden change of $\epsilon(T)$ and $p(T)$ above the critical temperature, $G_{0}$ and $G_{2}$ show an abrupt decrease around $T_{c}$.

As a further point, we have to evaluate the temperature dependence of the dimension 6 condensate, in order to obtain a consistent estimate of the temperature dependence of Eq.(3). To do this, we make use of a relation based on the dilute instanton gas model, giving

$$
\left\langle g^{3} f^{a b c} G_{\mu}^{a v} G_{v}^{b \lambda} G_{\lambda}^{c \mu}\right\rangle=\frac{48 \pi^{2}}{5 \rho_{c}^{2}}\left\langle\frac{\alpha_{s}}{\pi} G^{2}\right\rangle,
$$

where $\rho_{c}$ is the instanton radius, for which we use $\rho_{c}=0.33 \mathrm{fm}$ at $T=0$ [25]. We now assume that the above relation also holds at finite temperature and furthermore take into account the change of $\rho_{c}$ at finite $T$ [26], to obtain an estimate of the dimension 6 gluon condensate at $T=0$. Its effect on the behavior of the charmonium states below or above $T_{c}$ however turns out to be small, therefore even though the above considerations are quite crude, their details will not influence the results of this study.

\section{The Maximum Entropy Method}

In this subsection, the main ideas of the MEM procedure are briefly reviewed. For more details, see $[17,27,28]$.

The kind of problem that one aims to solve with the help of MEM is the following. One is interested in some function $\rho(\omega)$, but has only information about an integral of $\rho(\omega)$ :

$$
G_{\mathrm{OPE}}(M)=\int_{0}^{\infty} d \omega K(M, \omega) \rho(\omega),
$$

where $K(M, \omega)$ is the kernel and corresponds to

$$
K(M, \omega)=2 \omega e^{-\omega^{2} / M^{2}}
$$

in the case of the sum rules studied in this article. If $G_{\mathrm{OPE}}(M)$ is known only with limited accuracy or is only calculable in a limited range of the Borel mass $M$, the problem of obtaining $\rho(\omega)$ from $G_{\mathrm{OPE}}(M)$ is ill-posed and will not be analytically solvable.

The MEM approach now uses Bayes' theorem, by which additional information about $\rho(\omega)$ such as positivity and/or its asymptotic behavior at small or large energies can be included into the analysis in a systematic way and by which one can finally deduce the most probable from of $\rho(\omega)$. Bayes' theorem can be expressed as

$$
P[\rho \mid G H]=\frac{P[G \mid \rho H] P[\rho \mid H]}{P[G \mid H]},
$$

where the prior knowledge about $\rho(\omega)$ is denoted as $H$ and $P[\rho \mid G H]$ stands for the conditional probability of $\rho(\omega)$ given $G_{\mathrm{OPE}}(M)$ and $H$. Dropping the constant term $P[G \mid H]$ in the denominator because 


\section{EPJ Web of Conferences}

it does not depend on $\rho(\omega)$ and maximizing the remaining functional will give the most probable $\rho(\omega)$. $P[G \mid \rho H]$ is the "likelihood function" and can be constructed as

$$
\begin{aligned}
P[G \mid \rho H] & =e^{-L[\rho]}, \\
L[\rho] & =\frac{1}{2\left(M_{\max }-M_{\min }\right)} \int_{M_{\min }}^{M_{\max }} d M \frac{\left[G_{\mathrm{OPE}}(M)-G_{\rho}(M)\right]^{2}}{\sigma^{2}(M)} .
\end{aligned}
$$

$G_{\mathrm{OPE}}(M)$ is obtained from the OPE of the two-point function and $G_{\rho}(M)$ is defined as on the right hand side of Eq.(6). and therefore implicitly depends on $\rho(\omega)$. The function $\sigma(M)$ describes the uncertainty of $G_{\mathrm{OPE}}(M)$ at the corresponding value of the Borel mass.

On the other hand, $P[\rho \mid H]$ is called the "prior probability" and is given as

$$
\begin{aligned}
P[\rho \mid H] & =e^{\alpha S[\rho]}, \\
S[\rho] & =\int_{0}^{\infty} d \omega\left[\rho(\omega)-m(\omega)-\rho(\omega) \log \left(\frac{\rho(\omega)}{m(\omega)}\right)\right],
\end{aligned}
$$

where $S[\rho]$ is called the Shannon-Jaynes entropy. The function $m(\omega)$, introduced in Eq.(10) is the "default model". In the case of no available data $G_{\mathrm{OPE}}(M)$, the MEM procedure will just give $m(\omega)$ for $\rho(\omega)$ because this function maximizes $P[\rho \mid H]$. The default model can be used to incorporate known information about $\rho(\omega)$ into the calculation.

Assembling the functions given above, we get the final form for the probability $P[\rho \mid G H]$ :

$$
\begin{aligned}
P[\rho \mid G H] & \propto P[G \mid \rho H] P[\rho \mid H] \\
& =e^{Q[\rho]}, \\
Q[\rho] & \equiv \alpha S[\rho]-L[\rho] .
\end{aligned}
$$

At this point, it is only a numerical problem to obtain the form of $\rho(\omega)$ that maximizes $Q[\rho]$ and is the most probable $\rho(\omega)$ given $G_{\mathrm{OPE}}(M)$ and $H$. For this, we use the Bryan algorithm [29], which is widely used in MEM studies.

Once we have found $\rho_{\alpha}(\omega)$ which maximizes $Q[\rho]$ for a fixed value of $\alpha$, this parameter is integrated out by averaging $\rho(\omega)$ over a range of values of $\alpha$. The explicit procedure for this step is explained in [17]. The result this last integration then leads to our final result $\rho_{\text {out }}(\omega)$.

\section{Results}

We will in this article study the behavior of the charmonium ground states in the pseudoscalar and vector channels. First, we briefly describe our choices of the various parameters. In the actual analysis, we use the dimensionless quantity $v$ instead of the Borel mass $M$, so we first discuss the region of this parameter used in the analysis. The upper boundary of the region of $v$ to be analyzed is determined by the criterion that the dimension 6 term should be smaller than 20\% of the whole OPE expression of Eq.(3), which gives $v_{\max }^{\mathrm{V}}=8.04$ in the vector and $v_{\max }^{\mathrm{PS}}=7.29$ in the pseudoscalar channel. These values are kept fixed when going to finite temperature. As a matter of fact, in the temperature region around $T_{c}$, the relative contribution of the dimension 6 term at $v_{\max }^{\mathrm{V}, \mathrm{PS}}$ is even smaller, namely around $10 \%$ or less, and therefore does not much influence the results. Meanwhile, we choose the lower boundary of $v$ to be $v_{\min }^{\mathrm{V}, \mathrm{PS}}=0.78$, corresponding to a Borel mass of $M=3.0 \mathrm{GeV}$, and have checked that the obtained spectral functions do not depend much on this choice. For the value of the charm quark mass $m_{c}$, we take $\bar{m}_{c}\left(m_{c}\right)=1.277 \pm 0.026 \mathrm{GeV}$ obtained in [30], for $\alpha_{s}$ the newest world average $\alpha_{s}\left(M_{Z}\right)=0.1184 \pm 0.0007$ is employed [31], while for the vacuum gluon condensate $G_{0}^{\mathrm{vac}}$ the standard value $G_{0}^{\mathrm{vac}}=0.012 \pm 0.0036 \mathrm{GeV}^{4}[10,32]$ is used. The default model $m(\omega)$ is fixed to a constant matched to the perturbative value of the spectral function at high energy, similar to studies using lattice QCD [6]. 


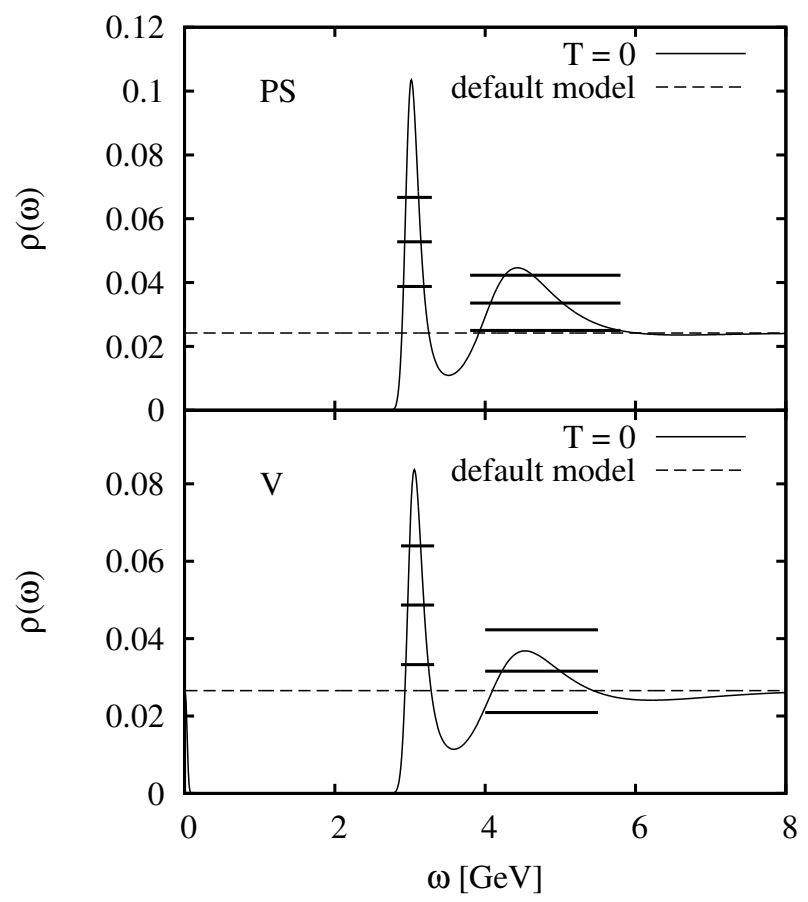

Fig. 1. The spectral functions in the pseudoscalar (upper plot) and vector (lower plot) channel at $T=0$, with the errors of their averaged values are shown. The horizontal extent and position of the error bars indicates the region over which the average is taken. The dashed lines show the default model used in the MEM analysis.

Let us now first examine the analysis results at $T=0$. The obtained spectral functions are shown in Fig. 1. In both channels, the ground state peaks corresponding to $\eta_{c}$ and $J / \psi$ are clearly extracted. The $\eta_{c}$ peak lies at $3.02 \mathrm{GeV}$, while the one representing $J / \psi$ is located at $3.06 \mathrm{GeV}$. We can thus observe that while the peak positions reproduce the experimental values with a precision of about 50 $\mathrm{MeV}$, the hyperfine splitting is underestimated. These findings are in agreement with earlier QCD sum rule studies [21], showing that the MEM analysis works properly. It also has to be mentioned that we observe broad second peaks in both channels. Comparing with an analysis of mock data, we conclude that these peaks originate from a combination of the continuum and several resonances, which cannot be resolved by the MEM analysis. Furthermore, it is observed that in the vector channel, the spectral function rises sharply at $\omega=0$. This is an artifact, caused by our usage of the derivative sum rules in this channel, which can be understood as follows. When taking the derivative, the sum rule looses all its information of the spectral function at $\omega=0$. Therefore, the MEM procedure will just approach the default model at this point, which is what we see in Fig. 1.

As a next step, we increase the temperature, following Eqs. (3) and (4). The obtained spectral functions in hot media between $T=0.9 T_{c}$ and $T=1.2 T_{c}$ are given in Fig. 2. We clearly observe a sudden change in the spectral function just above $T_{c}$, which is related to the abrupt increase of $\epsilon(T)$ and $p(T)$ at this temperature. The detailed behavior of the spectral function can be summarized as follows: first, the peaks experience a shift of about $50 \mathrm{MeV}$ to lower energies and then suddenly "melt" into the continuum. For $\eta_{c}$, the melting temperature is $T=1.0 T_{c}$, while for $J / \psi$ it lies slightly higher, namely at $T=1.1 T_{c}$. The shift of the peak observed here is perfectly consistent with the previous QCD sum rule analysis which used a more conventional analysis method [13-16].

We would like to mention here that, unlike in similar studies using lattice $\mathrm{QCD}$, the number of data points that can be used for the MEM analysis are not reduced when the temperature is increased, allowing a reliable extraction of the spectral function at finite temperatures. Moreover, we have checked that the additional uncertainties that enter into the analysis at finite temperatures due to the functions $\epsilon(T)$ and $p(T)$ do not cause a substantial reduction of the peaks. Thus, we conclude that the disap- 


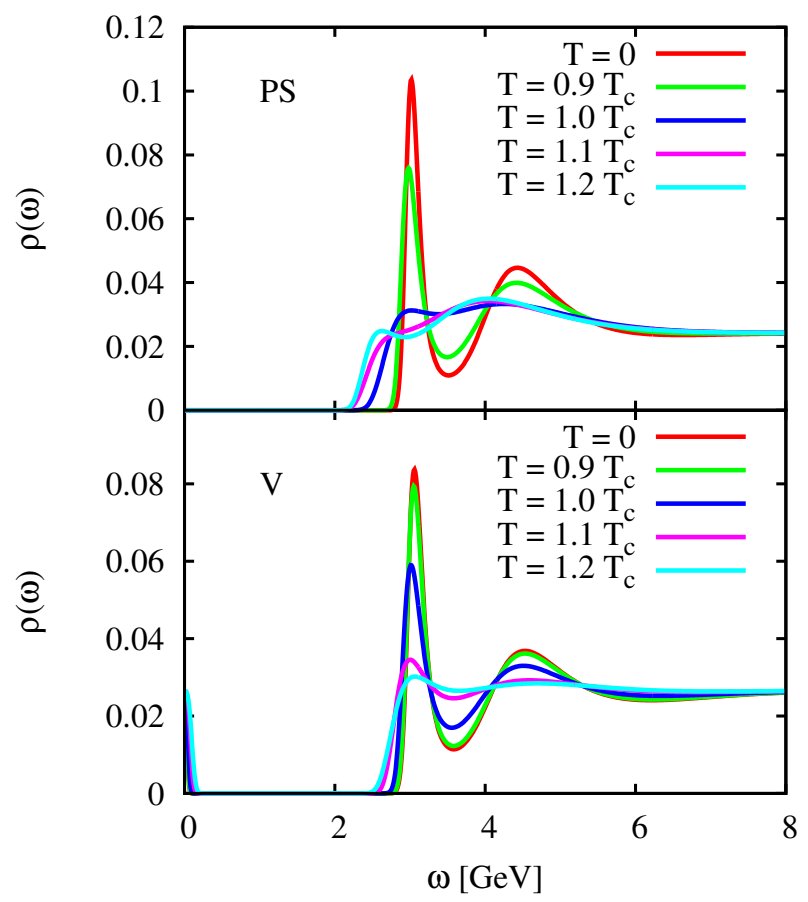

Fig. 2. The spectral functions in the pseudoscalar (upper plot) and vector (lower plot) channel at temperatures between $T=0.9 T_{c}$ and $T=1.2 T_{c}$. Here, the same default model as shown in Fig. 1 was used.

pearance of the peaks shown in Fig. 2 is a physical effect and not caused by an artifact of the MEM analysis.

\section{Conclusions}

We have in this study analyzed charmonia ground states at finite temperature in the vector- and pseudoscalar-channels. By combining the techniques of QCD sum rules and MEM, we have extracted the spectral functions from the OPE of correlators calculated in the deep Euclidean region. The OPE contains all the terms up to dimension 4 gluon condensates and dimension 6 scalar gluon condensate as an index of convergence. The values of the dimension 4 condensates at finite temperature were obtained from lattice QCD. As a result of the sudden shift of these values around $T_{c}$, we find that the ground state peaks dissolve into to continuum just above the critical temperature, which is in quantitative disagreement to the corresponding melting temperature observed in earlier lattice calculations of correlators at Euclidean time [6]. It, however, has to be remarked that our results are in fact consistent with the latest lattice results [33], finding the peaks of $\eta_{c}$ and $J / \psi$ to be largely distorted between 0.73 $T_{c}$ and $1.46 T_{c}$. It remains to be seen whether the difference of the melting temperature is of physical importance, or if it will disappear when both methods will become more accurate in the future.

\section{Acknowledgments}

This work was supported by KAKENHI under Contract Nos. 22105503, 19540275 and by YIPQS at the Yukawa Institute for Theoretical Physics. P.G. gratefully acknowledges the support by the Japan Society for the Promotion of Science for Young Scientists (Contract No. 21.8079). K.M. thanks for the support by FIAS. 


\section{References}

1. V. A. Novikov et al., Phys. Rept. 41, 1 (1978).

2. T. Matsui and H. Satz, Phys. Lett. B 178, 416 (1986).

3. T. Hashimoto, O. Miyamura, K. Hirose and T. Kanki, Phys. Rev. Lett. 57, 2123 (1986).

4. R. Rapp, D. Blaschke and P. Crochet, Prog. Part. Nucl. Phys. 65, 209 (2010).

5. H. Satz, J. Phys. G: Nucl. Part. Phys. 3225 (2006).

6. M. Asakawa and T. Hatsuda, Phys. Rev. Lett. 92, 012001 (2004).

7. S. Datta et al., Phys. Rev. D 69, 094507 (2004).

8. T. Umeda , K. Nomura and H. Matsufuru, Eur. Phys. J. C 39, 9 (2005).

9. A. Jacovác et al., Phys. Rev. D 75, 014506 (2007).

10. M.A. Shifman, A.I. Vainshtein and V.I. Zakharov, Nucl. Phys. B147, 385 (1979); B147, 448 (1979).

11. A.I. Bochkarev and M.E. Shaposhnikov, Nucl. Phys. B268, 220 (1986).

12. T. Hatsuda, Y. Koike and S.H. Lee, Nucl. Phys. B394, 221 (1993).

13. K. Morita and S.H. Lee, Phys. Rev. Lett. 100, 022301 (2008).

14. K. Morita and S.H. Lee, Phys. Rev. C 77, 064904 (2008).

15. Y. Song, S.H. Lee and K. Morita, Phys. Rev. C 79, 014907 (2009).

16. K. Morita and S.H. Lee, Phys. Rev. D 82, 054008 (2010).

17. P. Gubler and M. Oka, Prog. Theor. Phys. 124, 995 (2010).

18. K. Ohtani, P. Gubler and M. Oka, arXiv:1104.5577 [hep-ph].

19. P. Gubler, K. Morita and M. Oka, arXiv:1104.4436 [hep-ph].

20. R.A. Bertlmann, Nucl. Phys. B204, 387 (1982).

21. J. Marrow, J. Parker and G. Shaw, Z. Phys. C 37, 103 (1987).

22. T. Umeda, Phys. Rev D 75, 094502 (2007).

23. G. Boyd et al., Nucl. Phys. B469, 419 (1996).

24. O. Kaczmarek et al., Phys. Rev. D 70, 074505 (2004).

25. T. Schafer and E.V. Shuryak, Rev. Mod. Phys. 70, 323 (1998).

26. M.-C. Chu and S. Schramm, Phys. Rev. D 51, 4580 (1995).

27. M. Jarrel and J.E. Gubernatis, Phys. Rep. 269, 133 (1996).

28. M. Asakawa, T. Hatsuda, and Y. Nakahara, Prog. Part. Nucl. Phys. 46, 459 (2001).

29. R.K. Bryan, Eur. Biophys. J. 18, 165 (1990).

30. B. Dehnadi et al., arXiv:1102.2264 [hep-ph].

31. S. Bethke, Eur. Phys. J. C 64, 689 (2009).

32. P. Colangelo and A. Khodjamirian, "At the Frontier of Particle Physics/Handbook of QCD" (World Scientific, Singapore, 2001), Volume 3, 1495.

33. H.-T. Ding et al., PoS LAT2010, 180 (2010). 